\title{
An optimal RSSI-based cluster-head selection for sensor networks
}

\section{Khushboo Jain* and Anoop Kumar}

\author{
Department of CSE, \\ Banasthali Vidyapith, \\ Tonk, Rajasthan, India \\ Email: khushboojain2806@gmail.com \\ Email: anupbhola@banasthali.in \\ *Corresponding author
}

\begin{abstract}
Wireless sensor networks (WSNs) are becoming a very auspicious key to better address reliability to constant, accurate environmental monitoring. Although energy utilisation is one of the most common challenges, frequent communication between the sensor nodes (SNs) results in a huge energy drain. Moreover, load balancing and optimisation within the WSN are the significant factors for the extensive period of network lifetime. In fact, many such networks are deployed and operating outdoors is exposed to varying environmental conditions, which may further set grounds for severe performance degradation. Therefore, it is necessary to take into consideration factors like radio signal strength index to reduce the impact and to adapt to varying environmental conditions. In this paper, we propose an optimal RSSI $\mathrm{CH}$ selection (ORCHS) algorithm that is also based on environmental conditions to achieve energy efficiency and enhanced network lifetime by effectively managing energy levels within the network.
\end{abstract}

Keywords: cluster head selection; energy efficiency; network lifetime; residual energy; RSSI; wireless sensor networks; WSNs; node death rate; ContikiOS; ORCHS.

Reference to this paper should be made as follows: Jain, K. and Kumar, A. (2019) 'An optimal RSSI-based cluster-head selection for sensor networks', Int. J. Adaptive and Innovative Systems, Vol. 2, No. 4, pp.349-361.

Biographical notes: Khushboo Jain is a PhD Research Scholar in Computer Science Engineering at Banasthali Vidyapith, Tonk-Rajasthan India. She is pursuing her $\mathrm{PhD}$ in the area of 'Wireless Sensor Networks'. She received the MTech degree from Banasthali Vidyapith and BTech degree from UPTU Lucknow, India. She has published more than eight research papers as her contribution which has been published in various international journals of repute. Her research interests include wireless sensor networks, data prediction, data aggregation and data mining.

Anoop Kumar obtained his PhD from Banasthali Vidyapith, Rajasthan. He has done his Master's in Computer Applications (MCA) from MDU, Rohtak and is also a CCNA Certified Instructor. He is a member of renowned bodies like IEEE, CSI and Indian, Science National Congress. He has an extensive experience of more than 12 years in both teaching and academia. He is currently working as an Assistant Professor of Computer Science, Engineering and IT Department, Banasthali Vidyapith (Rajasthan). He has more than 30 research papers to his contribution which have been published in various national and international journals of repute. He is amongst one of the paper 
reviewers for reputed journals like IEEE, Springer, etc. He has been guiding several international and national computer science $\mathrm{PhD}$ and MTech scholars in the areas networking, clouding computing.

\section{Introduction}

Wireless sensor networks (WSNs) are becoming progressively a ubiquitous mechanism to monitor over a large geographical area for event detection and continuous monitoring applications (Bhola et al., 2017). The WSN gather the sensed value to intend the surroundings with further intellect. Its application are military and civil applications including environmental monitoring, home automation, surveillance and reconnaissance, fault analysis and diagnosis, precision agriculture, bio-medical applications, portfolio control, entity tracking. Its applications are also essential for disaster management like forest fire detection, tsunami warning, earthquake monitoring, landslide warning, weather forecasting and air and water quality monitoring. Due to their placement in hard-to-reach locations, it is usually problematic and expensive to or recharging or replacing batteries is not possible in most of the cases. Therefore, the recent trend in WSN is to develop energy-harvesting techniques that utilise ambient energy to elongate the network lifetime of sensor nodes (SNs) (Gupta and Sikka, 2015).

The SNs can be organised into clusters, which is a topological control technique to reduce activity of SN's transceivers. In each cluster, one or more $\mathrm{CHs}$ are selected to manage the activities within that cluster. There are three subsequent tasks that a $\mathrm{CH}$ has to perform: The first job is to collect sensed value from the SNs of that cluster regularly and aggregates them in-order eradicate duplicity that may exist between the correlated data. The succeeding job of $\mathrm{CH}$ is to create a time division multiple access (TDMA) schedule by which a SN can get a time slot in order to sends its data. The third job of the $\mathrm{CH}$ is to send aggregated values (Jain and Bhola, 2018) directly to the BS. If a fixed SN performs the entire three tasks, its lifetime will be of very short span. Thus, there is a need to change $\mathrm{CH}$ regularly and effectively.

In this work, we have proposed an optimal received signal strength index (RSSI) $\mathrm{CH}$ selection scheme, which is based on the residual energy of the SNs and the signal strength between the SNs and BS of that cluster. The proposed algorithm specifies the dynamicity of the $\mathrm{CH}$ and considers factors like radio signal strength for the section of the $\mathrm{CH}$. It has been obvious that RSSI values are significantly affected by environment and multi-path (mp) fading. RSSI values changes with the change in environmental conditions and also with the effect of time domain and frequency domain. RSSI values discrepancy and its strength do not relate with each other but they are individually depended on the environment complexity ( $\mathrm{Wu}$ et al., 2008). RSSI readings are predictable by estimating and measuring the radio signal strength and the majority of IEEE 802.11 and 802.15.4 RF modules sustain for its assessment and measurement.

The rest of the paper is organised as follows: Section 2 describes the related work and motivation, Section 3 presents the network model, energy model and our proposed algorithm (optimal received signal strength index $\mathrm{CH}$ selection - ORCHS), respectively. Section 4 presents the simulation and performance evaluation followed by the results. Finally, the paper is concluded in Section 5. 


\section{Related work and motivation}

Clustering which is a topological control technique plays an imperative role in energy management in the sensor network. Selecting a $\mathrm{CH}$ can suitably balance load and effectively manage data transmission by reducing energy depletion and improving sensor network lifetime. In this section, we present the valuable prior research accomplished in clustering protocols that are mainly determined towards energy efficiency and thereby improves overall network lifetime.

A cluster-based energy efficient forwarding scheme is presented in Adeel et al. (2010) based on binary exponential back off algorithm. This scheme ensures that whenever several SNs of a cluster receive a data packet, then only one SN among them is nominated to send the acceptance. Bajaber and Awan (2011) have proposed an adaptive decentralised re-clustering protocol (ADRP) which selects a $\mathrm{CH}$ and few $\mathrm{CHs}$ for subsequent rounds based on residual energy of each SNs and average energy of cluster. In this work, the energy utilisation criteria for re-clustering is avoided for next few rounds but $\mathrm{SN}$ death from next $\mathrm{CH}$ list make the WSN un-balanced.

A scalable energy efficient clustering hierarchy $(\mathrm{SEECH})$ is proposed in Tarhani et al. (2014), which selects CHs and transmits separately on the based on SN suitability. In this algorithm high degree SNs are classified and CHs and low degree SNs are employed as relays. SEECH uses a new distance-based algorithm to deliberate uniformity of CHs to balance clusters. The proposed algorithm when is compared with LEACH and TCAC method depicts enhanced performance of SEECH protocol in terms of sensor network lifetime. Nayak and Devulapalli (2016) had addressed the issue of suitable $\mathrm{CH}$ selection. The proposed approach is implemented by using fussy logic with the standard LEACH protocol. The inputs to fussy inference system are the residue of energy level, location of BS and cluster's centrality. The performance of this approach was enhanced in terms of stability and network lifetime.

Singh et al. (2016) have presented HEED protocol with varying level heterogeneity. This enhanced HEED protocol is based on model parameters and demonstrates energy-efficiency with better throughput and increases the packet delivery to the BS. A new protocol energy efficient optimal chain protocol (EEOC) for increasing energy efficiency of WSN is proposed in Agarwal et al. (2016). The results of simulation is compared with LEACH, PEGASIS, and ACT, etc. and concluded that important performance measures are first node die (FND), HNA and LNA. EEOC outperforms the other protocols and ensure energy efficiency.

El Alami and Najid (2016) has proposed an EEFL-CH technique by improving the LEACH protocol in-order to reduce the energy utilisation by using Fuzzy logic-based technique. The enhanced protocol uses parameters like energy-efficiency, distance between SNs and BS and residue energy of SNs for cluster formation. Mehra et al. (2017) had proposed a fuzzy logic-based clustering algorithm for WSN (CAFL) which is an enhancement over CFFL. In this algorithm, they have chosen parameters like residue energy of $\mathrm{SNs}$ and proximity to $\mathrm{BS}$ for $\mathrm{CH}$ selection. For cluster formation they have chosen parameters like proximity to $\mathrm{CH}$ of SNs and residue energy of tentative $\mathrm{CH}$ is taken into concern for competence.

El Alami and Najid (2017) had presented a zones-based cluster formation in which the sensor field is divided into three equal-sized zones. The $\mathrm{CH}$ selection will be dynamic for providing load-balancing and uniform dissipation of energy by the deployed 
SNs. A simple yet effective clustering algorithm is presented in Padmanaban and Muthukumarasamy (2018) known as energy efficient structured clustering algorithm (EESCA). The algorithm uses average communication distance and remaining energy for selecting the CHs. Further the CHs are rotated based on the CTNR parameter. The analysis id performed on the basis of simulation time, load balancing, scalability, FND and CUDP. Simulation results are compared with LEACH and SEECH protocols and proved to be advantageous for environmental monitoring.

A priority-based weighted clustering algorithm for ad hoc networks presented in Pathak and Jain (2019). It uses three different stages to $\mathrm{CH}$ changes and clustering overhead. In first stage, the algorithm selects the CHs based on their initial priority of SN inside cluster. The second stage aids in cluster maintenance. In addition, in third stage the new $\mathrm{CH}$ is automatically selected without any delay when residual battery life of previous $\mathrm{CH}$ reaches to minimum threshold value. Experimentation and simulation of the proposed algorithm proved the validity of the work and confirms the theoretical claims. Zeng et al. (2019) have proposed an energy-coverage ratio clustering protocol (ECRCP) to minimise the energy depletion and to utilise the coverage ratio. They have designed an energy model, which determines optimal number of clusters on the principle of 'minimum energy depletion' and selects the $\mathrm{CH}$ on the on the principle of 'coverage maximisation'. Experimentation with the proposed model proved to attain elongated network lifetime, load balancing and lower energy consumption in the environment of heterogeneous sensor network.

Behera et al. (2019) focuses on an efficient $\mathrm{CH}$ selection algorithm, which rotates the $\mathrm{CH}$ position among the SNs with higher energy intensity as compared to other. The proposed scheme is based on the initial energy, residual energy and an optimum number of $\mathrm{CH}$ to select the next group of $\mathrm{CHs}$ for the network that is beneficial for IoT applications such as environmental monitoring, home automation, surveillance, fault analysis, precision agriculture. Experimentation proved that the proposed scheme outperforms the LEACH in terms of throughput, network lifetime and residual energy.

Jain et al. (2020) focus the research on improving sensor network lifetime. Instead of changing cluster heads for dynamic clustering at every round of communication, they develop an optimal $\mathrm{CH}$ threshold function and an energy threshold function to propose the dynamicity of cluster head based on the current energy intensities, thus improves the network's lifetime.

\section{Proposed approach}

This section consists of the network model, energy model and our proposed approach which is an ORCHS algorithm and is based on residual energy levels of the SNs and the signal strength of SNs to BS.

\subsection{Network model}

The proposed algorithm assumes a network consisting of $\mathrm{SNs}$ which are randomly distributed with uniform density in a geographical region of size $100 * 100 \mathrm{~m}^{2}$. After deployment the $\mathrm{SN}$ is restricted to change its physical location. This means that the coordinates of SN remains fixed throughout the network lifetime. A two-tier architecture is considered where $\mathrm{BS}$ is positioned in the centre of the $3 \mathrm{D}$ space surrounded by SNs. 
All SN are considered with equal energy and computational capabilities which may differ in their signal strength, i.e., RSSI value. BS has the responsibility to elect $\mathrm{CHs}$ in cluster formation phase and nearby nodes has a responsibility to join one of the elected $\mathrm{CH}$. The communication is considered as multi-hop communication where SN send their sensed data to their respective $\mathrm{CH}$ which in-turn aggregates all the received data send it to BS.

\subsection{Energy model}

There are usually four working system of a SN which are processing unit, sensing unit, radio communication subsystem which includes a receiver, transmitter, amplifier and antenna; and a energy unit. The energy dissipation in a SN is depended on the processing unit, sensing unit and radio unit of the SN. A simple model for the radio communication energy dissipation is assumed in which transmitter fritters energy to run the radio electronics (Heinzelman et al., 2002).

Figure 1 Radio dissipation model

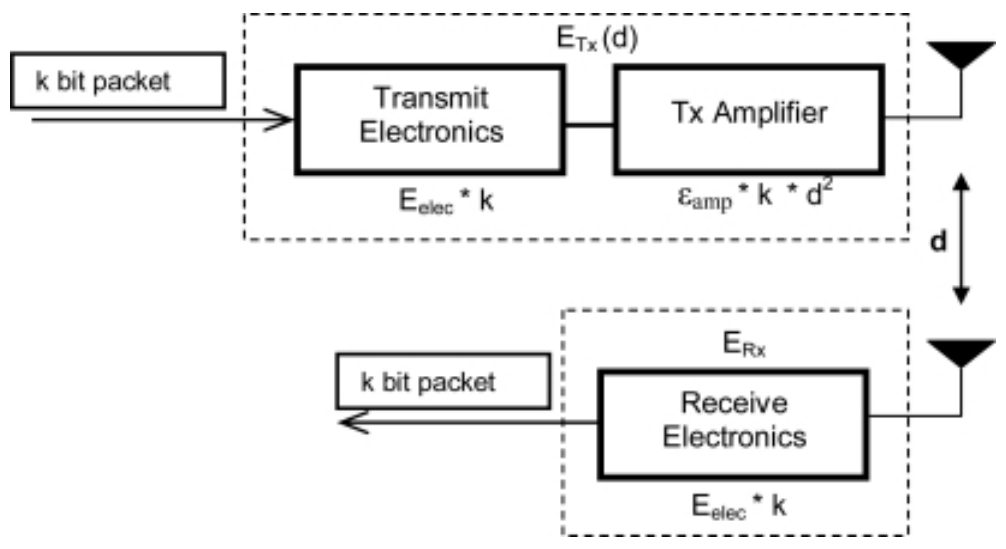

This model uses both free-space $\left(f_{s}\right)$ and $m p$ fading channels depending on the distance between the transmitter and receiver. A threshold is defined to determine the channel to be used. If the distance is less than the threshold, $f_{s}$ channel is used on the other hand if the distance is greater than the threshold; the mp channel is used (Heinzelman et al., 2002). Thus, to send a $k$-bit data packet over a distance $d$, the radio spends:

$$
\begin{aligned}
& E_{T X}(k, d)=E_{T X-\text { elec }}(k)+E_{T X-\text { amp }}(k, d) \\
& E_{T X}(k, d)= \begin{cases}E_{\text {elec }} * k+k \varepsilon_{f s} d^{2} & \text { if } d<d_{0} \\
E_{\text {elec }} * k+k \varepsilon_{m p} d^{4} & \text { if } d \geq d_{0}\end{cases}
\end{aligned}
$$

where threshold $d_{0}$ is given as

$$
d_{0}=\sqrt{\frac{\varepsilon_{f s}}{\varepsilon_{m p}}}
$$

To receive the data packet, the radio spends: 


$$
E_{R X}(k)=E_{R X-\text { elec }}(k)=k E_{\text {elec }}
$$

\subsection{ORCHS algorithm}

The proposed ORCHS algorithm has following three phases.

\subsubsection{Setup and cluster formation phase}

In this phase all $\mathrm{SN}$ will communicate with the $\mathrm{BS}$ for selection of $\mathrm{CHs}$ and cluster formation. Therefore, each SN broadcast an advertisement to the BS. From this advertisement the BS creates the vector array with SN ids and their respective RSSI value. The BS then chooses $\mathrm{CHs}$ on the basis of Maximum RSSI value. Thus the likelihood selecting a first $\mathrm{CHs}_{2} \mathrm{LC}_{-} H[i]$ are estimated from the following equation:

$$
L_{-} C H[i]=\sum_{i=0}^{n} \max (R[i])
$$

where $n$ is the number of SNs and $R[i]$ is the $i^{\text {th }}$ RSSI from the corresponding SN. Originally, energy of all SNs would be same, therefore the energy factor is not considered in the setup phase to select initial CHs. Now the BS broadcasts message to all SNs to inform about the initial $\mathrm{CHs}$, the $\mathrm{SN}$ in near proximity will transmit join-request message to $\mathrm{CH}$. The $\mathrm{CH}$ will acknowledge them accordingly.

\subsubsection{Data communication phase}

The BS broadcasts a message to all SNs to update details about the recently selected CHs to all clusters in the WSN. At this instant, a TDMA schedule is allocated by the $\mathrm{CH}$ to all SNs of that cluster. Each SN can transmit the sensed data to their respective $\mathrm{CH}$ in the time slot available to them. $\mathrm{CHs}$ will collect the data from SNs and aggregates them. The aggregated data will be then forwarded to the BS.

\subsubsection{CH re-election phase}

This phase determines the need of re-election of CHs. By experimentally analysing varying range of residual energy level of present $\mathrm{CH}$, the threshold value for switching the $\mathrm{CH}$ is estimated and is shown in the next section. The $\mathrm{CH}$ will broadcast $\mathrm{CH}$ ELECT to all SNs within that cluster, whenever the residual energy $E[i]$ of that $\mathrm{CH}$ drops to $40 \%$ of its initial energy. This constitute that the present $\mathrm{CH}$ is not having sufficient energy to perform the work of the $\mathrm{CH}$ and an instantaneous switching is in need for extending the lifetime of WSN. On receiving this broadcast each SN will sends a parameter $S[i]$ to $\mathrm{CH}$, which is calculated by the summing up of its residual energy level $E[i]$ and RSSI value $R[i]$ all SNs. Now the current $\mathrm{CH}$ will evaluate the threshold, $S_{-} C H[i]$ for selecting the next $\mathrm{CH}$. The threshold for selecting the next $\mathrm{CH}$ is estimated from the following equation:

$$
S_{-} C H[i]=\sum_{i=0}^{n}\left(\frac{(R[i]+E[i])}{n}\right)
$$


where $n$ represents the total $\mathrm{SNs}$ in that cluster. The present $\mathrm{CH}$ will now compares $S \_C H[i]$ with $S i$ value for each SN. The SN which has just maximum $S i$ value than the threshold $S_{-} C H[i]$ will be selected as the next $\mathrm{CH}$.

\section{Algorithm: ORCHS}

Procedure: Initialise: Construct vector array containing the following data for each $\mathrm{SN}$.

$V_{X}[i], V_{Y}[i], E[i], S[i]$, Type $[i], R[i], D[i], S N_{I D}[i]$

\section{Parameters:}

$1 V_{X}[i] \quad \leftarrow$ Vector of X-coordinate of SN

$2 V_{Y}[i] \quad \leftarrow$ Vector of Y-coordinate of SN

$3 E[i] \quad \leftarrow$ Energy of SNs and is initialised by E0

$4 S[i] \quad \leftarrow$ Status of each SN (died $=0$ or active $=1)$

$5 \quad$ Type $[i] \quad \leftarrow$ Type of $\mathrm{SN}($ normal $=0$ or $\mathrm{CH}=1$ )

$6 \quad R[i] \quad \leftarrow$ RSSI value of SNs

$7 D[i] \quad \leftarrow$ Distance from $\mathrm{BS} / \mathrm{CH}$

$8 S N_{I D}[i] \leftarrow$ Sensor node ID

Algorithm 1 Setup and cluster formation phase

1 Assign random position to $\mathrm{SN}$

2 Assign a central fixed location to BS.

3 Compute $D[i]$ of each SN from BS.

4 Send $R[i]$ value of each $\mathrm{SN}$ to $\mathrm{BS}$.

5 BS will create lookup-table with $S N_{I D}[i]$ and its $R[i]$ value

6 BS will choose $\mathrm{CH}$ depending upon max $R[i]$ value from equation (5) and acknowledge each $\mathrm{SN}$ with 0 or $1(\mathrm{SN}=0$ and $\mathrm{CH}=1)$

a if $\mathrm{SN}$ receive 1 , change Type $[i]=\mathrm{CH}$

b if $\mathrm{SN}$ receive 0 , change Type $[i]=\mathrm{SN}$

7 Each SN will send join-request message to its nearby cluster's $\mathrm{CH}$.

$8 \mathrm{CH}$ will acknowledge $\mathrm{SNs}$ by sending a tuple ( $S N_{-} i d$, Response).

a if Response $=1, \mathrm{SN}$ will belongs to that $\mathrm{CH}$

$\mathrm{b} \quad$ if Response $=0, \mathrm{SN}$ will not belongs to that $\mathrm{CH}$.

Algorithm 2 Data communication phase

1 All SNs will send the sensed data to their respective $\mathrm{CH}$ for each successive round of communication

2 Energy of a SN will change as:

a $E[i]=E[i]-E_{T X}$

$\left[\left(E_{T X}\right.\right.$ will be computed from equations (1), (2) and (3)]

$3 \mathrm{CH}$ upon receiving the sensed data will aggregate it.

4 Energy of a $\mathrm{CH}$ will change as:

a $E[i]=E[i]-E_{R X}$

[ $E_{R X}$ will be computed from equation (4)] 
Algorithm $3 \mathrm{CH}$ re-election phase

1 if $(E[C H]]<40 \%$ its initial energy)

a $\mathrm{CH}$ broadcast a CH-ELECT to BS and all SNs belongs to that cluster

2 else

a Continue to be $\mathrm{CH}$.

3 Upon receiving CH-ELECT, SNs of that cluster will send their $R[i]$ and $E[i]$ to the $\mathrm{CH}$

$4 \mathrm{CH}$ will elect the new $\mathrm{CH}$ by calculating and comparing $S \_C H[i]$ value of each SN with $S[i]$

5 SN with just higher $S[i]$ value than $S_{-} C H[i]$ will be then selected as a new $\mathrm{CH}$.

$6 \mathrm{CH}$ acknowledge each $\mathrm{SN}$ with 0 or $1(\mathrm{SN}=0$ and $\mathrm{CH}=1)$

$7 \mathrm{CH}$ change its Type $[i]=\mathrm{CH}$ and other $\mathrm{SN}$ will receive 0 and changes their Type $[i]=\mathrm{SN}$.

\section{Simulations and performance evaluations}

This section describes the simulation environment and performance metrics that we have used to analyse ORCHS algorithm. We have also stated the investigational analysis to select threshold for $\mathrm{CH}$ selection. Finally, we have presented the results and demonstrate the proficiency analysis of our ORCHS approach.

\subsection{Simulation environment and performance metrics}

\subsubsection{Simulator used}

ContikiOS (Dunkels et al., 2004) is open source operating system for resource constraint hardware devices with low power and less memory. It supports a variety of motes like MicaZ, sky motes, Z1 mote, etc. Contiki provides Cooja simulator (Österlind et al., 2006) to simulate the network of SN. We can choose the type of node from any of these three categories: Cooja node, Java node and emulated nodes. Cooja provides simple GUI for easier deployment and configuration of SN. Cooja is a cross level simulator which provides flexibility and extensibility in all levels. As the SNs in WSN are provided with radio frequency which has capabilities of both transmission and receiving a data packet, the RSSI value can be used to approximate the communication distance between the SNs and BS. A total of $100 \mathrm{SN}$ were simulated with varying cluster size $(5,10,20$ and 25) in an area of $100 \mathrm{~m} * 100 \mathrm{~m}$.

\subsubsection{Performance metrics}

1 Network lifetime: It is defined as the operational time of the network during which it is capable of performing its dedicated tasks. It can be divided into three categories: FND, half node die (HND) and last node die (LND).

2 Node death rate (NDR): It denotes the total number of live SN over the rounds. A lower NDR indicates that the load is balanced over the WSN. The region of NDR can be categorised as stable region and unstable region. All SN are alive in the stable region whereas the unstable region represents the rest of the region. 


\subsection{Investigational analysis to select the energy threshold for $\mathrm{CH}$ selection}

We have performed experiments to determine the energy threshold value for selecting the next $\mathrm{CH}$. The experiments were based on the residual energy level of the current round of $\mathrm{CH}$. We have tried to establish the relation between the percentage of $\mathrm{CH}$ 's residual energy and network lifetime to determine an optimum value of energy threshold. In these experiments, the $\mathrm{CH}$ is placed at the centre of $100 \mathrm{~m} * 100 \mathrm{~m}$ sensing field surrounded by $100 \mathrm{SNs}$. The initial energy of all SN is taken as $2 \mathrm{~J}$. So the total energy of WSN will be $200 \mathrm{~J}$.

In our analysis, we have vary the residual threshold energy for re-election of the CHs. We have performs for experiments with threshold ranging from $10 \%$ to $90 \%$ and fixed the cluster size to be 10 to determine the network lifetime. It can be seen from Figure 2 that if $10 \%$ of residual energy is taken as threshold, the lifetime of WSN will be about 1,152 rounds. On varying the residual energy percentage until $40 \%$, the WSN lifetime reaches to 1,431 rounds and if we vary residual energy level further the number of rounds decreases gradually. Therefore, we have taken threshold for selecting new $\mathrm{CH}$ to $40 \%$ and this value was used for calculation in $\mathrm{CH}$ re-election phase.

Figure 2 Selection for optimal threshold value for $\mathrm{CH}$

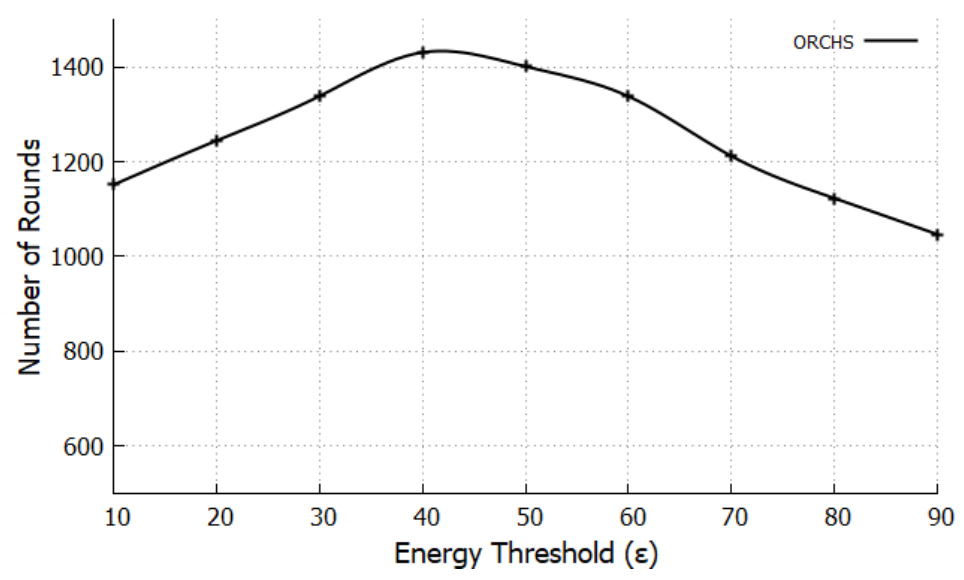

\subsection{Results and discussion}

To examine the performance of our ORCHS algorithm we have compared its performance with classical LEACH (Nayak and Devulapalli, 2016) and SEECH (Tarhani et al., 2014). The simulation parameters used during our experimentation for all approaches are mentioned in Table 1. The performance of our ORCHS algorithm was demonstrated on the basis of following metric: network lifetime (FND, HND and LND) and NDR. We have taken four cluster sizes $(5,10,20$ and 25) to analyse the impact of varying load on $\mathrm{CH}$ and to determine overall network lifetime (FND, HND and LND) in all the cases. Table 2 lists the variation in cluster size with a total network size of 100 SNs. 
Table 1 Simulation parameters

\begin{tabular}{lc}
\hline Parameter & Value \\
\hline WSN area & $100 \mathrm{~m} \times 100 \mathrm{~m}$ \\
Total no. of SNs & 100 \\
Protocols & LEACH, SEECH, ORCHS \\
BS location & $(50,50)$ \\
SN initial energy & $2 \mathrm{~J}$ \\
Total energy & $200 \mathrm{~J}$ \\
SN distribution & Random \\
Eelec (for $\mathrm{T}_{\mathrm{x}}$ and $\left.\mathrm{R}_{\mathrm{x}}\right)$ & $50 * 10^{-9} \mathrm{~J} / \mathrm{bit}$ \\
Eamp & $100 * 10^{-12} \mathrm{~J}$ \\
Aggregation energy & $5 \mathrm{~nJ} / \mathrm{bit} / \mathrm{s}$ \\
Packet header size & $25 \mathrm{bytes}$ \\
Data packet size & $100 \mathrm{bytes}$ \\
\hline
\end{tabular}

Table 2 FND, HND and LND value for various approaches

\begin{tabular}{lcccc}
\hline Protocol & Cluster size & $F N D$ & $H N D$ & LND \\
\hline LEACH & & 406 & 697 & 789 \\
SEECH & & 654 & 703 & 891 \\
ORCHS & 5 & 801 & 1,021 & 1,265 \\
ORCHS & 10 & 792 & 1,125 & 1,431 \\
ORCHS & 20 & 653 & 1,284 & 1,671 \\
ORCHS & 25 & 602 & 1,267 & 1,541 \\
\hline
\end{tabular}

The results of simulation that has been carried out to determine the network lifetime in terms of (FND, HND and LND) of different algorithms like LEACH, SEECH and our proposed ORCHS are represented in Figure 3. It is evident that the number of rounds of LEACH, SEECH and ORCHS with cluster size 5, 10, 20 and 25 take for FND found to be 406, 654, 801, 792, 653, 602 correspondingly. The ORCHS algorithm takes more rounds with all cluster sizes for FND in comparison to other approaches. Similarly the HND is $697,703,1,021,1,125,1,284$ and 1,267 correspondingly. The ORCHS algorithm takes more rounds with all cluster sizes for HND in comparison to other approaches. Correspondingly, the LND is 789, 891, 1,265, 1,431, 1,671 and 1,541 respectively. The ORCHS algorithm takes more rounds with all cluster sizes for LND in comparison to other approaches.

Figure 4 shows the comparison between LEACH, SEECH and ORCHS in term of NDR. It is partitioned into two regions: one is stable region and other is unstable region. In stable region, all the SNs are alive and as the SNs starts dying region become unstable. In this figure we have plotted total WSN energy against each round. It is evident from results that the NDR of our approach is always less than that of LEACH and SEECH. 
Figure 3 Network lifetime

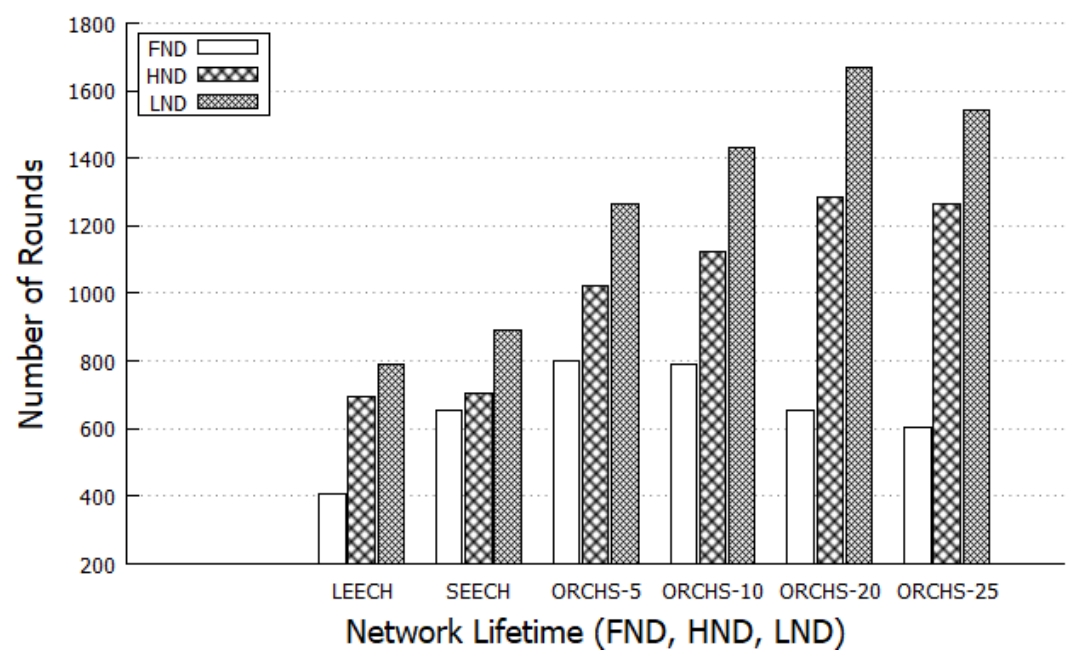

Figure 4 Node death rate

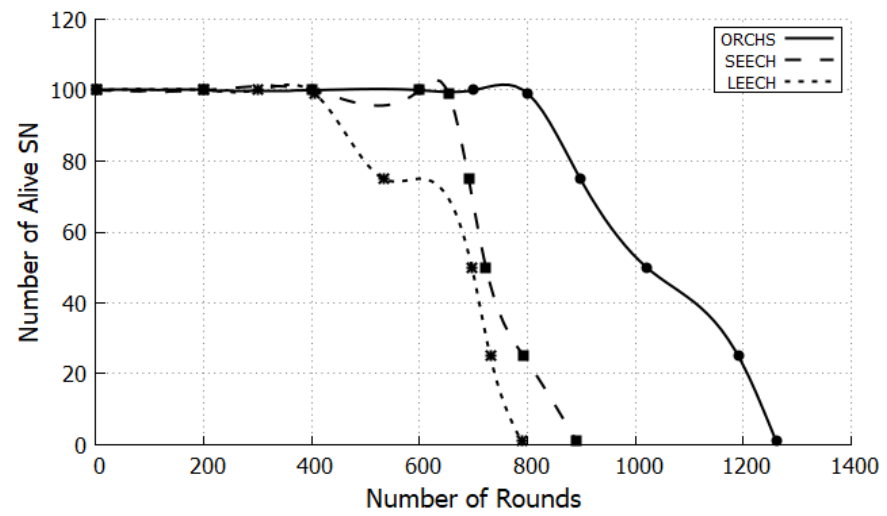

On the basis of above analysis and results, it has been apparently confirmed that ORCHS ensures higher energy efficiency and prolonged network lifetime.

\section{Conclusions and future aspect}

In order to improve energy efficiency and enhance network lifetime of sensor network, we have proposed and simulated an optimal RSSI CH selection ORCHS approach. The ORCHS has uniform energy utilisation and thus it has more residual energy available for a large number of rounds. It reduces communications overheads and avoids unnecessary selection of CHs after every round of communication. Simulation results also asserted that our proposed algorithm has prolonged network lifetime as compared to LEACH and SEECH. The algorithm has considerably achieved $35 \%$ to $45 \%$ and $30 \%$ to $35 \%$ efficiency in terms of energy preservation when compared to LEACH and SEECH 
respectively and the number of rounds has increased from around 789 to 1,671 in case of LEACH and from 891 to 1,671 in case of SEECH. Future work will be dedicated to inculcate scalability in the proposed work.

\section{References}

Adeel, A., Ali, M.A. and Sohail, J. (2010) 'Energy aware intra cluster routing for wireless sensor networks', Int. J. Hybrid Inform. Technol., Vol. 3, No. 1, pp.29-48.

Agarwal, A., Gupta, K. and Yadav, K. (2016) 'A novel energy efficiency protocol for Wsn based on optimal chain routing', IEEE Xplore 2016 3rd Int Conf Comput Sustain Glob Dev, pp.488-493.

Bajaber, F. and Awan, I. (2011) 'Adaptive decentralized re-clustering protocol for wireless sensor networks', Journal of Computer System and Science, Vol. 77, No. 2, pp.282-292.

Behera, T.M., Mohapatra, S.K., Samal, U.C. et al. (2019) 'Residual energy-based cluster-head selection in WSNs for IoT application', IEEE Internet Things J., Vol. 6, pp.5132-5139, DOI: 10.1109/JIOT.2019.2897119.

Bhola, A., Mhatre, M. and Jha, C.K. (2017) 'Energy efficient WSN using membership handshaking clustering technique for isolated nodes', presented in First International Conference on Smart Technologies in Computer and Communication (SmartTech-2017).

Dunkels, A., Gronvall, B. and Voigt, T. (2004) 'Contik i - a lightweight and flexible operating system for tiny networked sensors', Proceedings of the 29th Annual IEEE International Conference on Local Computer Networks, pp.455-462.

El Alami, H. and Najid, A. (2016) 'Energy-efficient fuzzy logic cluster head selection in wireless sensor networks', in 2016 International Conference on Information Technology for Organizations Development (IT4OD), IEEE [online] https://doi.org/10.1109/IT4OD.2016. 7479300 .

El Alami, H. and Najid, A. (2017) 'Fuzzy logic based clustering algorithm for wireless sensor networks', Int. J. Fuzzy Syst. Appl., Vol. 6, pp.63-82 [online] https://doi.org/10.4018/IJFSA.2017100105.

Gupta, K. and Sikka, V. (2015) 'Design issues and challenges in wireless sensor networks', International Journal of Computer Applications, Vol. 112, No. 4, pp.26-32, February.

Heinzelman, W., Chandrakasan, A. and Balakrishnan, H. (2002) 'An application-specific protocol architecture for wireless micro sensor network', IEEE Transactions on Wireless Communications, Vol. 1, No. 4, pp.660-670 [online] http://anrg.usc.edu/contiki/index.php/ RSS_measurement.

Jain, K. and Bhola, A. (2018) 'Data aggregation design goals for monitoring data in wireless sensor networks', Journal of Network Security Computer Networks, Vol. 4, No. 3, pp.1-9 [online] http://doi.org/10.5281/zenodo.1464554.

Jain, K., Kumar, A. and Jha, C.K. (2020) 'Probabilistic-based energy-efficient single-hop clustering technique for sensor networks', in Bansal, J., Gupta, M., Sharma, H. and Agarwal, B. (Eds.): Communication and Intelligent Systems. ICCIS 2019. Lecture Notes in Networks and Systems, Vol. 120, Springer, Singapore.

Mehra, P.S., Doja, M.N. and Alam, B. (2017) 'Zonal based approach for clustering in heterogeneous WSN', Int. J. Inf. Technol., pp.1-9 [online] https://doi.org/10.1007/s41870017-0071-2.

Nayak, P. and Devulapalli, A. (2016) 'A fuzzy logic-based clustering algorithm for WSN to extend the network lifetime', IEEE Sens. J., Vol. 16, pp.137-144 [online] https://doi.org/10.1109/ JSEN.2015.2472970.

Österlind, F. et al. (2006) 'Cross-level sensor network simulation with COOJA', Proceedings of the First IEEE International Workshop on Practical Issues in Building Sensor Network Applications, Tampa, Florida, USA. 
Padmanaban, Y. and Muthukumarasamy, M. (2018) 'Energy-efficient clustering algorithm for structured wireless sensor networks', IET Networks, Vol. 7, pp.265-272, DOI: 10.1049/ietnet.2017.0112

Pathak, S. and Jain, S. (2019) 'A priority-based weighted clustering algorithm for mobile ad hoc network Sunil Pathak * and Sonal Jain', Int. J. Commun. Networks Distrib. Syst., Vol. 22, No. 3, pp.313-328.

Singh, S., Chand, S. and Kumar, B. (2016) 'Energy efficient clustering protocol using fuzzy logic for heterogeneous WSNs', Wirel. Pers. Commun., Vol. 86, pp.451-475 [online] https://doi. org/10.1007/s11277-015-2939-4.

Tarhani, M., Kavian, Y.S. and Siavoshi, S. (2014) 'SEECH: scalable energy efficient clustering hierarchy protocol in wireless sensor networks', IEEE Sens. J., Vol. 14, pp.3944-3954, DOI: 10.1109/JSEN.2014.2358567.

Wu, R-H., Lee, Y-H., Tseng, H-W., Jan, Y-G. and Chuang, M-H. (2008) 'Study of characteristics of RSSI signal', 2008 IEEE International Conference on Industrial Technology, Chengdu, pp.1-3, DOI: 10.1109/ICIT.2008.4608603.

Zeng, M., Huang, X., Zheng, B. and Fan, X. (2019) 'A heterogeneous energy wireless sensor network clustering protocol', Wirel. Commun. Mob. Comput., DOI: 10.1155/2019/7367281. 